\title{
Regelungskonzept für eine schadstoffarme Gasturbinenbrennkammer
}

\author{
Herrn Prof. Dr. rer.nat. Harro Kiendl, unserem Mitautor, zum 60. Geburtstag gewidmet
}

Jürgen Adamy, Erlangen, Ulf Schwane und Harro Kiendl, Dortmund, Detlef Altemark, Dorsten, Achim Saul und Bernhard Adomeit, Essen Dr.-Ing. Jürgen Adamy ist Gruppenleiter im Forschungszentrum Erlangen der
Siemens AG. Hauptarbeitsfelder: Entwicklung und Realisierung regelungs-
technischer Verfahren, Fuzzy-Systeme, neuronale Netze, Modellbildung und
Simulation.
Adresse: Siemens AG, Forschungszentrum Erlangen, Bereich Automatisie-
rungstechnik, Abt. AUT 941, Paul-Gossen-Str.100, 91052 Erlangen, Tel.: (09131) 7-33124

Dipl.-Ing. UIf Schwane ist wissenschaftlicher Angestellter am Lehrstuhl für Elektrische Steuerung und Regelung (Prof. Dr. rer. nat. H. Kiendl) der Universität Dortmund. Hauptarbeitsfelder: Adaptive Regelungssysteme für Mehrarmroboter, Regelungskonzepte für Erdgas-Verdichteranlagen (Stations-, Gasturbinen- und Pumpverhütungs-Regelung).

Adresse: Universität Dortmund, Fakultät für Elektrotechnik, Lehrstuhl für Elektrische Steuerung und Regelung, 44221 Dortmund, Tel.: (02 31) 755-3592, Email: uetx09@zx2.hrz.uni-dortmund.de

Prof. Dr. rer. nat. Harro Kiendl ist Leiter des Lehrstuhls für Elektrische Steuerung und Regelung der Universität Dortmund. Hauptarbeitsfelder: Nichtlineare und robuste Regelungssysteme, klassische und regelbasierte Modellierung, Fuzzy Control (Entwurf, Stabilität und Inferenzstrategien).

Adresse: siehe oben, Tel.: (0231) 755-2760.

Dr.-Ing. Detlef Altemark ist Leiter der Ảbteilung Industrielle Gasanwendung bei der Ruhrgas AG, Betriebsstelle Dorsten. Hauptarbeitsfelder: Entwicklung der industriellen Gasanwendung auf den Gebieten der Feuerungs- und Verbrennungstechnik.

Adresse: Ruhrgas Aktiengesellschaft, Huttropstr. 60, 45138 Essen, Tel. (02362) 93-8503.

Dr.-Ing. Achim Saul ist Leiter der Abteilung Kommerzielle Projekte Ost bei der Ruhrgas AG, Essen. Hauptarbeitsfelder: Nach der Entwicklungstätigkeit in der industriellen Gasanwendung über Taätigkeiten im Technischen Controlling werden heute kommerzielle Aktivitäten des Unternehmens in Osteuropa betreut.

Adresse: Ruhrgas Aktiengesellschaft, Huttropstr. 60, 45138 Essen.

Dipl.-Ing. Bernhard Adomeit ist Leiter der Abteilung Anlagenautomationstechnik bei der Ruhrgas AG, Essen. Hauptarbeitsfelder: Automatisierung von Prozessèn aufi ferngesteuerten Speichèr- und Verdichterstationen für den Erdgasferntransport.

Adresse: Ruhrgas Aktiengesellschaft, Huttropstr. 60, 45138 Essen.

Den Gasturbinen wurde wegen ihres wachsenden Marktanteils in den letzten Jahren besondere Aufmerksamkeit geschenkt. Neben der Verbesserung des Wirkungsgrades wurden Entwicklungen zur Minimierung der Schadstoffemissionen vorangetrieben. Das Minderungspotential ist bei der Verwendung von Brenngasen der offentlichen Gasversorgung besonders hoch. Während bei konventionellen Brennkammern eine Regelung der Verbrennung nicht notwendig ist, sind zur vollen Ausnutzung des Schadstoffreduktionspotentials und für einen sicheren und zuverlässigen Betrieb der Gasturb̈ine neue Regelungskonzepte erforderlich. In dieser Arbeit wird ein Regelungskonzept für eine extrem schadstoffarme Gasturbinenbrennkammer für den Betrieb mit Erdgas vorgestellt. Die Regelung ist als Temperaturregelung konzipiert und.den speziellen Anforderungen des Gasturbinenbetriebs angepaßt. Dabei wird die meßtechnisch schwer erfaßbare Flammentemperatur aus der gemessenen Luftzahl ( $\lambda$-Wert) unter Annahme eines adiabaten Verbrennungsablaufs mittels der Wärmebilanz berechnet.

Als Grundlage für den Entwurf des Regelungssystems wird für den Brenner und die angrenzenden Prozesse ein Modell erstellt. Das Regelungskonzept basiert auf drei wesentlichen Komponenten: Erstens wird die Zufuhr von Verbrennungsluft schnell, aber ungenau vorgesteuert und unterlagert nachgeregelt. Zweitens wird die Genauigkeit durch eine langsame überlagerte Temperaturregelung verbessert. Drittens wird die Genauigkeit durch eine Adaption weiter gesteigert. Die Effizienz des Regelungskonzeptes wird anhand von Simulationen sowie anhand von Versuchen an einem Brennerprototyp demonstriert.

\section{Control concept for a low-pollutant gas turbine combustion chamber}

Particular attention has been paid to the gas turbine in recent years due to its growing share of the market. In addition to improvements in efficiency, there have also been further developments to reduce pollutant emissions. The potential here is particularly high with fuel from the public gas supply. Whereas combustion control is not necessary with conventional combustion chambers, new control concepts are in demand if gas turbines are to be safely and reliably operated and if the potential for reducing pollutants is to be fully exploited. This paper presents a control concept for an extremely low-pollutant, gas turbine combusion chamber using natural gas. The temperature control system has been adapted to the special requirements imposed by gas turbine operation. For this, the difficult measurable flame temperature is calculated from the measured air-coefficient variable ( $\lambda$-value) by means of a thermal balance assuming an adiabatic combustion.

A model was prepared for the burner and adjacent processes as a basis for the design of the control system. The concept is based on three major components: Firstly, the supply of combustion air is precontrolled rapidly, but inaccurately and subsequently subjected to underlayed 
control. Secondly, the accuracy of this control system is improved by a slow higherlevel temperature control. Thirdly, accuracy is improved still further by means of adaptation. The efficiency of the control concept is demonstrated with the use of simulations and trials on a burner prototype, repectively.

\section{Einführende Übersicht}

Beim Überlandtransport großer Mengen Erdgas in Pipelines werden alle $200 \mathrm{~km}$ Verdichterstationen benötigt, um den Druck des Erdgases, der während des Transports durch Reibung absinkt, wieder zu erhöhen. Dazu werden Turbokompressoren eingesetzt, die von Gasturbinen angetrieben werden. Für den Betrieb der Gasturbine wird Erdgas aus der Pipeline entnommen, so daß die Stationen kostengünstig und autark betrieben werden können. Sie sind damit ein wesentliches Element für die Versorgungssicherheit mit Erdgas. Um die natürlichen Umweltvorteile des Erdgases auch in Gasturbinen vollständig zu nutzen, kam es seitens der Gaswirtschaft in den letzten Jahren zu einer Forcierung der Entwicklung extrem schadstoffarmer Gasturbinenbrennkammern. Dies geschah zum einen, um diese Technik in den bereits beschriebenen Verdichterstationen für den Überlandtransport von Erdgas einzusetzen, zum anderen aber auch für Erdgaskunden, die eigene Gasturbinen betreiben.

Aufbau, Eigenschaften und Feuerungskonzepte von Gasturbinen werden in [1] näher beschrieben. Die Gasturbine besteht in ihrer einfachsten Ausführung aus Kompressor, Brennkammer und Turbine. Eine der möglichen Methoden zur Schadstoffminimierung bei der Verbrennung in der Gasturbinenbrennkammer ist die sogenannte Vormischtechnik [2]. Sie ist eine Primärmaßnahme, mit der die Schadstoffbildung bereits in der Flamme vermieden wird. Mit ihr werden sowohl extrem niedrige $\mathrm{NO}_{\mathbf{x}^{-}}$als auch $\mathrm{CO}$-Emissionen erreicht. Die Vormischtechnik ist ausschließlich für dampf- bzw. gasförmige Brennstoffe, z. B. Erdgas, geeignet, denn der Brennstoff muß mit der Verbrennungsluft bei hohem Luftüberschuß vollständig und möglichst homogen vermischt werden, bevor das Gemisch in den Brenner gelangt. Die Verfahrenstechnik dieser Brennkammern ist weitestgehend bekannt [3].

Während bei konventionellen Brennkammern entsprechend der geforderten Abtriebsleistung nur die Brennstoffmenge geregelt wird und sich dabei konstruktionsbedingt die zulässigen und erforderlichen Temperaturen einstellen, muß bei Vormischbrennern eine vorgegebene Verbrennungstemperatur, z.B. die adiabate Flammentemperatur, eingehalten werden. Die adiabate Flammentemperatur ist die maximale Temperatur in der Flamme, die im Falle vollständiger Vermischung bei gegebener Luftzahl, Brennstoffart und Eintrittstemperatur des Gemisches auftreten kann.

Es gibt erfahrungsgemäß ein Fenster der adiabaten Flammentemperatur, innerhalb dessen der Gasturbinenbrenner betrieben werden kann, so daß es bei verschiedenen Betriebszuständen weder zum Erlöschen der Flamme wegen Brennstoffmangels (zu niedrige Flammentemperatur) noch durch eine brennstoffreiche Verbrennung zu einer Überhitzung von Bauteilen und erhöhten $\mathrm{NO}_{\mathbf{x}}$-Emissionen aufgrund erhöhter Flammentemperaturen kommt. Zur Regelung der Verbrennung wird als Führungsgröße diejenige adiabate Flammentemperatur festgelegt, die einen zuverlässigen und sicheren Gasturbinenbetrieb, eine lange Lebensdauer sowie niedrige Schadstoffemissionen gewährleistet.

Temperturmessungen in der Flamme sind schon unter Laborbedingungen sehr schwierig und unzuverlässig und scheiden deshalb für eine Regelung der Verbrennung aus [4]. Daher wird als Ersatzregelgröße die leichter bestimmbare Luftzahl verwendet, die bei gegebenem Brennstoffi(Brenngasart) sowie Eintrittstemperaturen von Verbrennungsluft und Brenngas eindeutig der adiabaten Flammentemperatur durch die Wärmebilanz zugeordnet ist. Für die Bestimmung der Luftzahl stehen zuverlässige und bekannte Meßtechniken zur Verfügung.

Insgesamt soll die Regelung zwei Ansprüchen genügen. Zum einen soll sie die Flammentemperatur möglichst konstant halten, zum anderen soll sie auch schnelle zeitliche Änderungen, wie im Falle eines Lastabwurfs, beherrschen.

Gegenstand dieser Arbeit ist ein Regelungskonzept, das diese Aufgabe löst. Zunächst wird der Gasturbinenbrenner beschrieben und ein Brennermodell erstellt. Danach wird das Regelungskonzept vorgestellt und seine Effizienz anhand von Simulationen und experimentellen Ergebnissen demonstriert.

\section{Der Gasturbinenbrenner}

Bei dem Gasturbinenbrenner handelt es sich um ein sogenanntes vorgemischtes System, d.h., Gas und Verbrennungsluft werden bereits vor der Verbrennung homogen miteinander vermischt. Lokale Temperaturinseln und hierdurch bedingte hohe $\mathrm{NO}_{\mathbf{x}}$-Konzentrationen, wie sie bei heterogenen Gemischen oder Direkt= einspritzung des Gases in die Brennkammer entstehen, werden so vermieden [5].

Die durch einen Kompressor verdichtete Verbrennungsluft wird durch ein Rohrsystem über ein Ventil $V_{\boldsymbol{V}}$ (mit dem der Volumenstrom einstellbar ist) dem Mischer zugeführt und dort mit dem einströmenden Gas vermischt (Bild 1). Das entstandene Gemisch wird durch eine gelochte Brennerplatte in die Brennerkugel geleitet und dort gezündet. Die Verbrennung findet in der Brennkammer statt. Das Abgas wird mit Kühlluft vermischt und der Turbine zugeführt. Die Kühlluft dient einerseits der Kühlung der Flammenschutzhülle, um eine Überhitzung durch die Flamme zu vemeiden, andererseits der Einstellung der Turbineneintrittstemperatur. Der Verbrennungs- und der Kühlluftmassenstrom werden von demselben Kompressor über ein ringförmiges Rohr geliefert. Die Aufteilung erfolgt erst im Brennersystem. Das Aufteilungsverhältnis wird durch die Ventile $V_{V}$ und $V_{K}$ bestimmt. In der Regel öffnet man $V_{K}$ vollständig und stellt den Verbrennungs- 


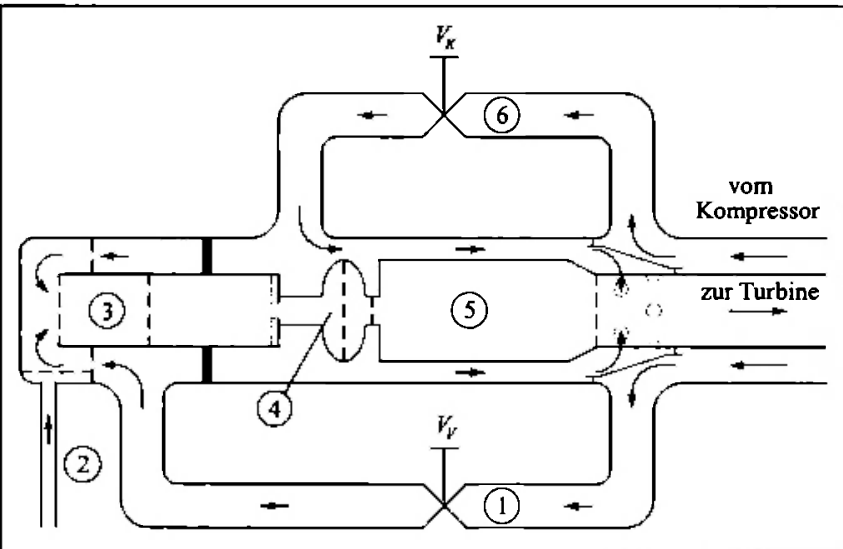

Bild 1: Aufbau des Brenners:
(1) Verbrennungsluft
(4) Brennerkugel (Zündung)
(2) Gas
(5) Brennkammer mit Flammenschutzhülle
(3) Mischer
(B) Kühlluft

luftmassenstrom $\dot{m}_{V}$ mittels $V_{V}$ ein. Nur wenn $V_{V}$ vollständig geöffnet ist und $m_{V}$ weiter erhöht werden soll, beginnt man, $V_{K}$ zu schließen.

Der Verbrennungsluftmassenstrom $\dot{m}_{V}$ wird so eingestellt, daß der Brenner überstöchiometrisch verbrennt, d.h., es wird mehr Luft in die Brennkammer eingebracht als tatsächlich zur Verbrennung benötigt wird. Ein Maß für den Überschuß ist die Luftzahl

$$
\tilde{\lambda}=\frac{\dot{m}_{V}}{\dot{m}_{\mathrm{sl}}}
$$

die das Verhältnis zwischen der für die Verbrennung zur Verfügung stehenden Luft $\dot{m}_{V}$ und der für die stöchiometrische, d.h. vollständige, Verbrennung benötigten Luft $\dot{m}_{\text {st }}$ angibt. Da im Falle $\lambda>1$ die nicht für die Verbrennung benötigte Luft die Flamme kühlt, kann über $\dot{m}_{V}$ als Stellgröße die Flammentemperatur beeinflußt werden.

\section{Das Prozeßmodell}

Das gesamte Brennersystem besteht im wesentlichen aus vier Teilsystemen: den Rohrleitungen mit den Ventilen, den Ventilantrieben, dem Mischer und der Brenn= kammer. Neben dem Brenner selbst ist für die Auslegung der Regelung auch die Dynamik der $\lambda$-Messung von Bedeutung. Alle Systeme werden im folgenden modelliert.

\subsection{Brennkammer}

Die Vorgänge in der Brennkammer lassen sich durch ihre Energiebilanz beschreiben. Aus dem 1. Hauptsatz der Thermodynamik für stationäre Fließprozesse [6] ergibt sich:

$$
\begin{aligned}
Q= & \left(\dot{m}_{G}+\dot{m}_{\mathrm{s} 1}\right) \cdot h_{A}\left(T_{A}\right)+\left(\dot{m}_{V}-\dot{m}_{\mathrm{st}}\right) \cdot h_{L}\left(T_{A}\right)- \\
& -\dot{m}_{G} \cdot h_{G}\left(T_{G}\right)-\dot{m}_{V} \cdot h_{L}\left(T_{L}\right)
\end{aligned}
$$

(vgl. Bild 2). Hierin bezeichnen $Q$ den abgeführten Wiärmestrom, $\dot{m}$ die Massenströme, $T$ die jeweilige Temperatur und $h(T)$ die zugehörige spezifische Enthalpie. Die Indizes $L, G$ und $A$ stehen für Luft, Gas und Abgas. Mit der Näherung

$$
\Delta h=h\left(T_{2}\right)-h\left(T_{1}\right) \approx c_{p} \cdot\left(T_{2}-T_{1}\right),
$$

wobei $c_{p}$ die mittlere spezifische Wärmekapazität bei konstantem Druck im Intervall $\left[T_{1}, T_{2}\right]$ ist, und dem unteren Heizwert ${ }^{1}$

$$
\begin{aligned}
\Delta h_{u}= & h_{G}(273.15 \mathrm{~K})+L_{\min } \cdot h_{L}(273.15 \mathrm{~K})- \\
& -\left(1+L_{\min }\right) \cdot h_{A}(273.15 \mathrm{~K}), \quad L_{\min }=\frac{\dot{m}_{\mathrm{sl}}}{m_{c}},
\end{aligned}
$$

erhält man aus (2) für adiabate Bedingungen, d.h. $\dot{Q}=0$ :

$T_{A}=\frac{\lambda \cdot L_{\min } \cdot c_{p, L}\left(T_{L}\right) \cdot T_{L}+c_{p, G} \cdot T_{G}+\Delta h_{u}}{\left(1+L_{\min }\right) \cdot c_{p, A}+(\lambda-1) \cdot L_{\min } \cdot c_{D . L}\left(T_{A}\right)}$

Diese Beziehung zeigt, daß die adiabate Flammentemperatur $T_{A}$ von den Prozeßgrößen $T_{L}, T_{G}$ und von

$$
\lambda=\frac{\dot{m}_{V}}{\dot{m}_{G} \cdot L_{\min }}
$$

abhängig ist.

\subsection{Mischer}

Der Mischer besteht aus einem System von Rohren, das von den einzelnen Gasmolekülen durchlaufen wird. Wenn man Wellenphänomene vernachlässigt, kann die Durchströmung des Mischers im einfachsten Fall durch ein Totzeitglied charakterisiert werden. Die Totzeit

\begin{tabular}{|c|c|c|c|}
\hline $\begin{array}{c}\text { Luft } \\
\left(\dot{m}_{\nu}, c_{p L}\left(T_{L}\right), T_{L}\right)\end{array}$ & \multirow[b]{2}{*}{ Brennkammer } & $\begin{array}{l}\text { Verbrennungsprodukte } \\
\left(\dot{m}_{G}+\dot{m}_{s t}, c_{p \cdot A}, T_{A}\right)\end{array}$ & \multirow{3}{*}{ Abgas } \\
\hline \multirow[t]{2}{*}{$\underset{\left(\dot{m}_{G}, c_{p, G}, T_{G}\right)}{\text { Gas }}$} & & $\stackrel{\text { Abluft }}{\left(\dot{m}_{V}=\dot{m}_{s t}, c_{p L}\left(T_{A}\right), T_{A}\right)}$ & \\
\hline & \multicolumn{2}{|c|}{ Abwärme $\dot{Q}$} & \\
\hline
\end{tabular}

$$
\tau_{M}=\frac{V_{M} \cdot \varrho_{M}}{\dot{m}_{G}+\dot{m}_{\mathrm{V}}}
$$

ergibt sich aus dem Volumen $V_{M}$ des Mischers, der Gemischdichte $\varrho_{M}$ und dem Gemischmassenstrom $\dot{m}_{G}+\dot{m}_{V}$.
Bild 2: Massen= und Wärmestrőme der Brennkammer.

\subsection{Rohrleitungen und Ventile}

Genauso wie der Mischer weist auch das Rohrsystem, durch das die Verbrennungsluft $\dot{m}_{V}$ strömt, eine Totzeit $\tau_{R}$ auf: Sie kann ähnlich wie oben nach (4) bestimmt werden.

\footnotetext{
1 Der untere Heizwert ist die freiwerdende spezifische Wărmemenge $\left(\mathrm{J} \cdot \mathrm{kg}^{-1}\right)$ einer Reaktion, bei der die zugeführten Reaktionsstoffe und die Reaktionsprodukte die gleiche Temperatur haben.
} 
Der Verbrennungsluftmassenstrom $\dot{m}_{V}$ wird durch die Öffnungsgrade $s_{V}$ und $s_{K}$ der Ventile $V_{V}$ und $V_{K}$ verändert. Hierbei ist zu beachten, daß $s_{V}$ und $s_{K}$ jeweils sowohl den Kühlluftmassenstrom $m_{K}$ als auch den Verbrennungsluftmassenstrom $\dot{m}_{V}$ beeinflussen. Bei der Modellierung läßt sich ausnutzen, daß die Strömungsgeschwindigkeit $v$ erheblich kleiner als die Schallgeschwindigkeit ist und deshalb in sehr guter Näherung die Strömungsgesetze für inkompressible Flüssigkeiten gelten [7]. Andererseits ist $v$ aber auch so groß, daß die Strömung turbulent ist. Daher gilt für den Druckabfall bei einer Rohrleitung mit der Länge $l$ und dem Radius $r$ [8]

$$
\Delta p=\frac{\chi \cdot l}{4 \pi^{2} \varrho_{L^{\prime}}{ }^{s}} \cdot \dot{m}^{2}=\frac{\zeta}{\varrho_{L}} \cdot m^{2}, \quad \zeta=\frac{\chi \cdot l}{4 \pi^{2} r^{5}} .
$$

Hierbei ist $\chi$ der dimensionale Reibungsbeiwert der Rohrwandung und $\varrho_{L}$ die Luftdichte. Für die Ventile gilt wie beim Rohr

$$
\Delta p=\frac{\zeta(s)}{\varrho_{L}} \cdot m^{2},
$$

wobei die geometrieabhängige Widerstandszahl ${ }^{2} \zeta(s)$ hier mit dem Öffnungsgrad $s$ variiert.

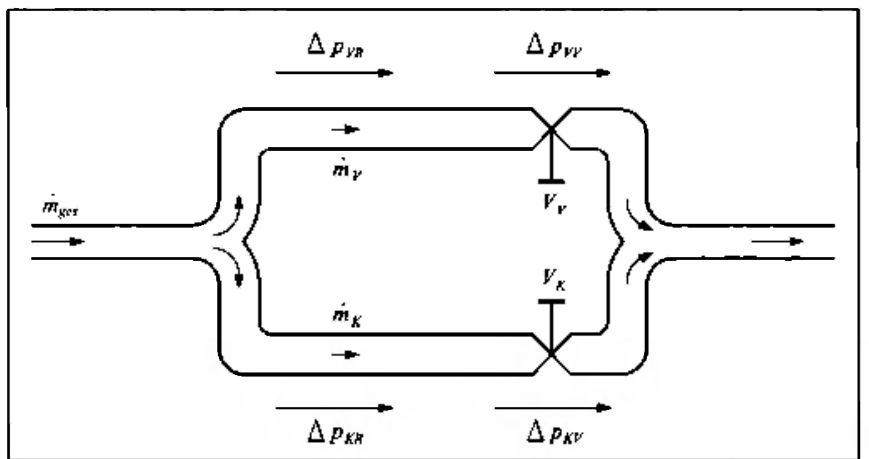

Bild 3: Massennströmê und Druckabfalle des Rohrsystems.

Nach Bild 3 teilt sich der gesamte Druckabfall auf in den des Ventils $\Delta p_{K V}$ und den des Rohres $\Delta p_{K R}$ des Kühlluftzweiges sowie in den des Ventils $\Delta p_{V V}$ und den des Rohres (einschließlich Brenner) $\Delta p_{V R}$ des Verbrennungsluftzweiges. Hierbei gilt

$$
\Delta p_{K V}+\Delta p_{K R}=\Delta p_{V V}+\Delta p_{V R} .
$$

Mit $\dot{m}_{\text {ges }}=\dot{m}_{K}+\dot{m}_{V}$ sowie (5) und (6) ergibt sich hieraus die Beziehung

$$
\dot{m}_{\mathrm{\nu}}=\frac{1}{1+\sqrt{\frac{\zeta_{V R}+\zeta_{V V}\left(s_{V}\right)}{\zeta_{K R}+\zeta_{K V}\left(s_{K}\right)}}} \cdot \dot{m}_{\mathrm{zes}},
$$

die die Abhängigkeit des Stromes $\dot{m}_{V}$ von den Ventilstellungen $s_{V}$ und $s_{K}$ zeigt. Wie oben beschrieben, werden die Ventile nicht unabhängig voneinander ver-

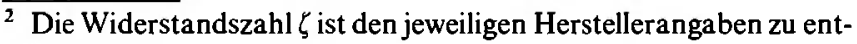
nehmen.
}

stellt: $V_{K}$ ist im allgemeinen voll geöffnet und wird nur variiert, wenn $V_{V}$ seinen maximalen Öffnungsgrad erreicht (Ablöseregelung). Die Übertragungscharakteristik (8) entspricht bei gegebenem $m_{\text {ges }}$ einer stark nichtlinearen Kennlinie, die bei der Berechnung der Sollwerte $s_{V \text {. soll }}$ und $s_{K \text {. soll }}$ für die Stellmechanismen von $V_{V}$ bzw. $V_{\mathrm{K}} \mathrm{zu}$ kompensieren ist.

\subsection{Stellantriebe der Ventile}

Zur Bewegung der Ventile im Verbrennungs- bzw. im Kühlluftzweig werden jeweils servohydraulische Rotationsantriebe verwendet, die einen identischen konstruktiven Aufbau aufweisen (Bild 4). Hauptelement eines solchen Hydraulikantriebs ist ein Servozylinder mit einseitig herausgeführter Kolbenstange, der die hydraulische Druckenergie in eine translatorische Bewegung umwandelt. Die Linearbewegung der Kolbenstange wird über ein Getriebe in eine Rotationsbewegung umgesetzt und dreht so den auf einer Welle sitzenden Kugelhahn im Luftventil. Wird die Lastankopplung ebenso wie die Zylinderaufhängung als starr angenommen, können alle bewegten Massen auf eine Gesamtmasse $m_{\text {red }}$ reduziert werden. Zur Steuerung des Antriebs dient ein Servoventil, das den Ölfluß in die Zylinderkammern bestimmt. Durch Anlegen einer elektrischen Spannung $U$ an den Torque-Motor des Servoventils kann die Ventilschieberposition $Y_{V}$ verändert werden, so daß eine der Kammern im Servozylinder mit dem Systemdruck $p_{s}$ und die andere Kammer mit dem Tankdruck $p_{0}$ verbunden wird.

Das dynamische Verhalten der Servoventile der vorliegenden Baureihe läßt sich in guter Näherung durch ein $P-T_{1}$-Glied beschreiben [9]:

$$
T_{V} \cdot Y_{V}+Y_{V}=K_{V} \cdot U
$$

Hierbei ist $T_{V}$ die Verzögerungszeitkonstante und $K_{V}$ der Übertragungsbeiwert der ersten Stufe des Servoventils. Für die Gültigkeit von (9) ist u. a. vorauszusetzen, daß exakte Nullüberdeckung der Steuerkanten des Ventilschiebers vorliegt und Reibung und Lose am Ventilschieber vernachlässigbar sind.

Bild 4: Schemaskizze des Servoantriebs.

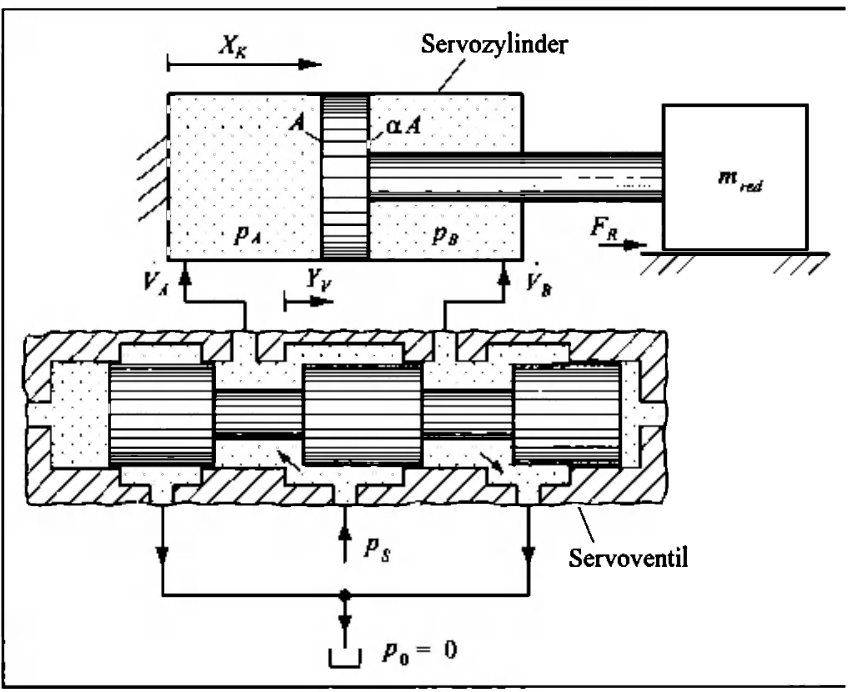


Die beiden Volumenströme $\dot{V}_{A}$ und $\dot{V}_{B}$ an den Steuerkanten des Ventilschiebers lassen sich mit Hilfe der Durchflußgleichung für Blenden nach Bernoulli berechnen [10]. $\mathrm{Zu}$ berücksichtigen ist dabei, daß das Vorzeichen von $Y_{V}$ die Strömungsrichtung von $V_{A}$ und $V_{B}$ sowie den Druckabfall festlegt. Die DurchfluBgleichungen nehmen somit die Gestalt

bzw.

$$
V_{A}=K_{Q} \cdot Y_{V} \cdot \sqrt{\left|\frac{p_{S}}{2}\left(1+\operatorname{sgn} Y_{V}\right)-p_{A}\right|}
$$

$$
\dot{V}_{B}=-K_{Q} \cdot Y_{V} \cdot \sqrt{\left|\frac{p_{S}}{2}\left(1-\operatorname{sgn} Y_{V}\right)-p_{B}\right|}
$$

an, wobei $K_{Q}$ die Volumenstromverstärkung des Servoventils ist und $p_{A}$ und $p_{B}$ die Kammerdrücke bezeichnen.

Die Volumenströme $\dot{V}_{A}$ und $\dot{V}_{B}$ können im Zylinder nicht unmittelbar zu einer Kolbenbewegung führen, weil unterschiedliche Einflüsse die Steifigkeit des Antriebes herabsetzen. Dabei spielt u.a. die Kompressibilität des Hydrauliköls eine wichtige Rolle. Definiert man daher eine hydraulische Kapazität $C_{h}$, die ein Maß für die Druckanstiegsgeschwindigkeit bei gegebenem Volumenstrom darstellt, ergeben sich unter Anwendung der Kontinuitätsgleichung die Bestimmungsgleichungen

$$
p_{A}=\frac{\dot{V}_{A}-A \cdot \dot{X}_{K}}{C_{h}}
$$

bzw.

$$
\dot{p}_{B}=\frac{\dot{V}_{R}+\alpha A \cdot \dot{X}_{K}}{C_{h}}
$$

für die Kammerdrücke.

Auf den Kolben wirken außer der von den Drücken $p_{A}$ und $p_{B}$ sowie ihren Angriffsflächen $A$ bzw. $\alpha A$ bestimmten Antriebskraft die Reaktionskraft der Last und die Reibkraft $F_{R}$. Das Verhalten des Kolbens wird durch die Newtonsche Bewegungsgleichung

$$
m_{\mathrm{red}} \cdot \ddot{X}_{K}=A \cdot p_{A}-\alpha A \cdot p_{B}-F_{R}
$$

beschrieben, wobei sich die Reibkraft

$$
F_{\mathbf{R}}=F_{C} \cdot \operatorname{sgn} \dot{X}_{K}+d \cdot \dot{X}_{K}
$$

aus einem richtungsabhängigen, trockenen (Coulombschen) Anteil und einem gesch windigkeitsproportionalen, viskosen (Newtonschen) Anteil zusammensetzt. Zudem kann ein Blockieren des Kolbens auftreten, nämlich dann, wenn die Kolbengeschwindigkeit einen Minimalwert $X_{\min }$ nahe Null unterschreitet und gleichzeitig die Antriebskraft die Haftreibkraft $F_{H}$ nicht überschreitet. Hieraus läßt sich die logische Blockierbedingung

$$
\left(\left|\dot{X}_{K}\right|<\dot{X}_{\min }\right) \wedge\left(\left|A \cdot p_{A}-\alpha A \cdot p_{B}\right|<F_{H}\right)
$$

ableiten. Durch das Blockieren des Kolbens wird die genaue Positionierung des Kolbens erschwert, und es stellt daher eine wichtige Antriebseigenschaft dar.
Eine weitere wichtige Antriebseigenschaft resultiert aus der Ungleichheit der beiden Kolbenflächen. Dadurch wird ein asymmetrisches Antriebsverhalten verursacht, das eine unterschiedliche maximale Geschwindigkeit beim Öffnen bzw. Schließen des Ventils mit sich bringt.

Letztlich sorgt das Getriebe über

$$
s=K_{G} \cdot X_{K}
$$

für eine proportionale Umsetzung der Kolbenposition in eine Ventilstellung $s$.

\section{5 $\lambda$-Messung}

Aus technischen Gründen erfolgt die $\lambda$-Messung nicht direkt am Brenner. Vielmehr wird dem Mischer ein kleiner, konstanter Volumenstrom $V_{\lambda}$ entnommen und über eine Leitung der Sonde zugeführt. Im Fall einer $\mathrm{ZrO}_{2}$-Sonde [11], wie sie hier verwendet wird, besitzt die Sonde ein konstantes Meßvolumen $V_{s}$, in dem die Verbrennung katalytisch, aber mit demselben Ergebnis wie im Brenner, erfolgt. Die Sonde bestimmt nun den $\lambda$-Wert nicht direkt, sondern sie ermittelt die $\mathrm{O}_{2}$-Volumenkonzentration der Verbrennungsprodukte. Der $\lambda$-Wert kann aus dieser Konzentration $\left[\mathrm{O}_{2}\right]$ errechnet werden. Hierzu berücksichtigt man, daß Luft zu $21 \%$ aus Sauerstoff besteht und Erdgas näherungsweise nur aus Methan, und erhält so die Rekationsgleichung

$\mathrm{CH}_{4}+(2+x) \mathrm{O}_{2}+(2+x) \cdot \frac{79}{21}$ Luft - ohne $-\mathrm{O}_{2} \rightarrow$
$\mathrm{CO}_{2}+2 \mathrm{H}_{2} \mathrm{O}+x \mathrm{O}_{2}+(2+x) \cdot \frac{79}{21}$ Luft - ohne $-\mathrm{O}_{2}$

wobei $x$ den Anteil unverbrannter Luft angibt. Aus dieser Reaktionsgleichung kann

$$
\lambda=\frac{2+x}{2}
$$

und

$$
\left[\mathrm{O}_{2}\right]=\frac{x}{3+x+(2+x) \cdot \frac{79}{21}}
$$

abgelesen werden. Aus (15) und (16) ergibt sich der Zusammenhang zwischen $\lambda$ und $\left[\mathrm{O}_{2}\right]$ :

$$
\lambda=\frac{0.21+0.105 \cdot\left[\mathrm{O}_{2}\right]}{0.21-\left[\mathrm{O}_{2}\right]} .
$$

Die Dynamik des obigen Meßvorgangs wird durch zwei Vorgänge bestimmt: zum einen durch das Totzeitverhalten der Leitung mit der Totzeit $\tau_{L}=V_{L} / \dot{V}_{\lambda}$ - hierbei ist $V_{L}$ das Leitungsvolumen -, zum anderen durch den Austauschvorgang des als Speicher wirkenden Sondenvolumens $V_{s}$. Innerhalb dieses Sondenvolumens gilt für die $\mathrm{O}_{2}$-Konzentration, die durch ein infinitesimal über die Leitung zugeführtes Volumen $d V_{\lambda}$ mit der Konzentration $\left[\mathrm{O}_{2}\right]_{\mathrm{zu}}$ verändert wird, der Mittelwert

$\left[\mathrm{O}_{2}\right]+d\left[O_{2}\right]=\frac{d V_{\lambda} \cdot\left[O_{2}\right]_{\mathrm{zu}}+\left(V_{S}-d V_{\lambda}\right) \cdot\left[\mathrm{O}_{2}\right]}{V_{S}}$. 
Hieraus folgt - nach Division durch $d t-$ das $\mathrm{P}_{-} \mathrm{T}_{1}-$ Verhalten

$$
\frac{V_{S}}{\dot{V}_{\lambda}} \cdot \frac{d\left[\mathrm{O}_{2}\right]}{d t}+\left[\mathrm{O}_{2}\right]=\left[\mathrm{O}_{2}\right]_{\mathrm{zu}} .
$$

Das gesamte Modell der $\lambda$-Messung besteht daher aus vier Teilen: Dies sind die Totzeit der Leitung, die Bestimmung der $\mathrm{O}_{2}$-Konzentration $\left[\mathrm{O}_{2}\right]_{\mathrm{zu}}$ mittels (1) und (17) zu

$$
\left[\mathrm{O}_{2}\right]_{\mathrm{zu}}=\frac{0.21 \cdot\left(\frac{\dot{m}_{V}}{\dot{m}_{G} \cdot L_{\min }}-1\right)}{0.105+\frac{\dot{m}_{V}}{\dot{m}_{G} \cdot L_{\min }}},
$$

die Sondendynamik (18) und schließlich die Berechnung des $\lambda$-Wertes aus $\left[\mathrm{O}_{2}\right]$ unter Verwendung von (17).

Das aus den obigen Teilmodellen der Abschnitte 3.1 bis 3.5 zusammengesetzte Modell bildet die Grundlage für den folgenden Regelungsentwurf.

\section{Regelungskonzept}

Die Regelung soll die adiabate Verbrennungstemperatur in der Brennkammer konstant halten. Liegt der $i$-Wert des Abgasgemisches als Meßgröße vor, kann die Verbrennungstemperatur anhand von (1) und (3) berechnet werden und steht daher als Regelgröße zur Verfügung.

Stelleingriff zur Beseitigung von Temperaturabweichungen ist der Verbrennungsluftmassenstrom $\dot{m}_{V}$. Dieser wiederum wird durch die Stellungen der Ventile $V_{V}$ und $V_{\mathrm{X}}$ bestimmt. Deshalb wird im Regelungskonzept eine kaskadenförmige Regelungsstruktur vorgesehen (Bild 5): Im Inneren sorgen die Lageregelkreise für die Positionierung der Ventile, diese werden von einem Durchflußregelkreis umschlossen, und dem Ganzen ist der Temperaturregelkreis überlagert. Diese Regelkreise werden im folgenden der Reihe nach beschrieben.

\subsection{Lageregelkreise der Ventile}

Die Lageregelkreise sorgen für eine schnelle Positionierung der Ventile, die sich in der Parallelschaltung von Kühl- und Verbrennungsluftzweig befinden. Für das oben entwickelte nichtlineare Modell der VentilStellantriebe läßt sich durch Linearisierung von (9) bis (14) im Arbeitspunkt $Y_{V}=0$ (,hydraulisch Null“) ein lineares Modell vierter Ordnung ableiten [12]. Dieses dient als Grundlage für den Entwurf der Lageregler. Dabei zeigt sich, daß bei den vorliegenden Antrieben bereits ein PD-T $\mathrm{T}_{1}$-Regler zu einem guten Systemverhalten führt (z. B. [13]).

\subsection{Durchflußregelkreis}

Die Durchflußregelung gibt die Stellungen der Ventile vor und damit nach (8) auch den Anteil der Gesamtluftmenge, der in die Brennkammer gelangt. Bei gegebener Gesamtluftmenge kann (8) für ein festes $s_{V}=s_{V, \text { max }}$ bzw. für ein festes $s_{K}=s_{K \text {. max }}$ abschnittsweise invertiert werden.

Mit den resultierenden, abschnittsweise definierten Gleichungen läßt sich das Stellsignal des dynamischen Durchflußreglers so umrechnen, daß zum einen die Ablöseregelung, zum anderen die Kompensation der nichtlinearen Kennlinie (8) realisiert wird.

Auf diese Weise kann man für den Entwurf des vorgesehenen PI-Durchflußreglers vereinfachend annehmen, daß immer nur einer der beiden Lageregelkreise aktiv ist und die Nichtlinearitäten vernachlässigbar sind, so daß sich die Reglerparameter z.B. mit Hilfe des Frequenzkennlinienverfahrens ermitteln lassen.

\subsection{Temperaturregelkreis}

Abweichungen in der Verbrennungstemperatur werden vom Temperaturregler beseitigt. Insbesondere die durch die lange Meßleitung zur $\lambda$-Sonde hervorgerufene Totzeit macht es unmöglich, allein über diese Temperaturregelung auf dynamische Änderungen des Gasmassenstromes schnell zu reagieren.

Daher liegt der Gedanke nahe, den Verbrennungsluftmassenstrom vorzusteuern, wodurch der Temperaturregler lediglich die auftretenden Störungen auszure-

Bild 5: Strukturbild der Regelung.

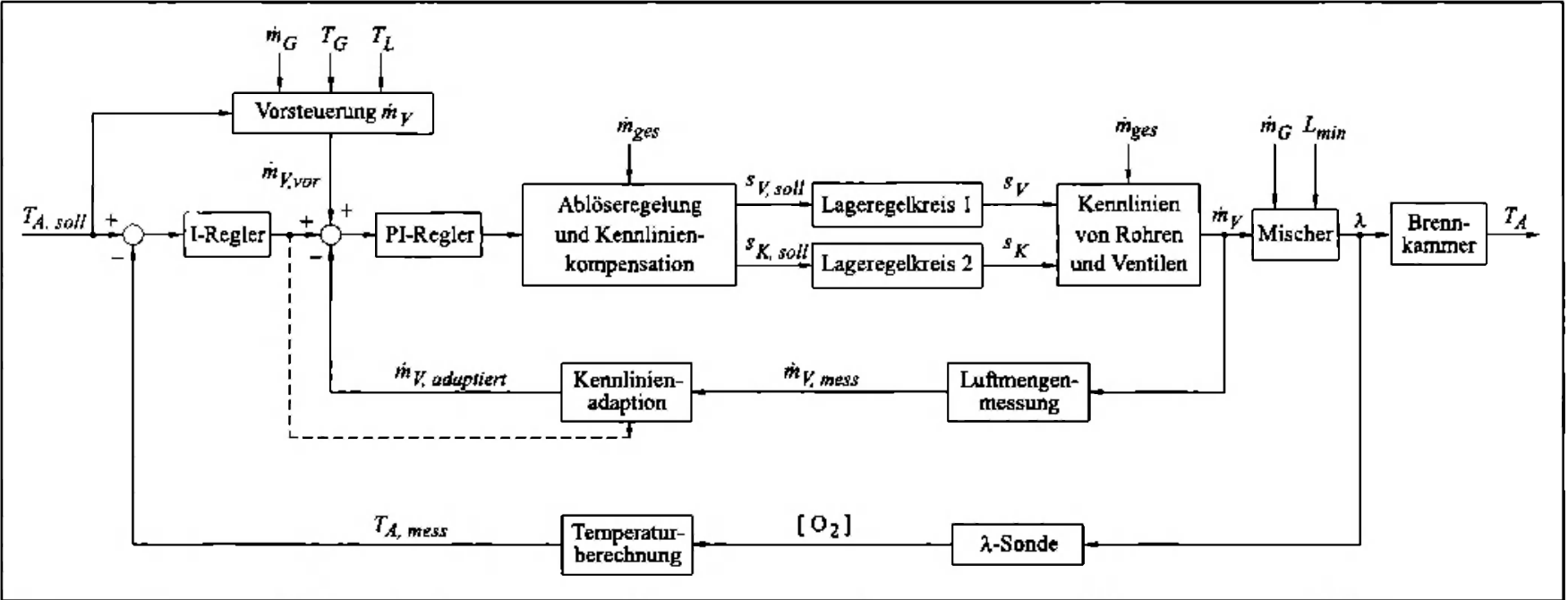


geln hat. Es läßt sich zeigen, daß dann bereits die Verwendung eines I-Reglers zu dem gewünschten Regelverhalten führt, so daß der Ausgang des I-Reglers dem einzustellenden Verbrennungsluftmassenstrom entspricht. Dessen Stellsignal kann dann für eine längerfristige Adaption der Durchflußmessung des Verbrennungsluftmassenstroms herangezogen werden, wodurch systematische Störgrößeneinflüsse ausgeglichen werden. Die Vorsteuerung des Verbrennungsluftmassenstroms und die Adaption der Durchflußmessung werden im weiteren kurz erläutert.

\subsection{Vorsteuerung des Verbrennungsluft- massenstroms}

Die umgeformte Energiebilanzgleichung (3) läßt sich bei gegebenem Gasmassenstrom mit (1) nach

$$
\begin{aligned}
& \dot{m}_{V, \text { vor }}= \\
& =\frac{\Delta h_{u}+c_{p, G} \cdot T_{G}-\left(\left(1+L_{\text {min }}\right) \cdot c_{p, A}-c_{p, L}\left(T_{A}\right)\right) \cdot T_{A, \text { soll }}}{c_{p, L}\left(T_{A}\right) \cdot T_{A, \text { soll }}-c_{p, L}\left(T_{L}\right) \cdot T_{L}}
\end{aligned}
$$

umstellen. Dies zeigt, daß sich der Verbrennungsluftmassenstrom bereits aufgrund der Kenntnis der Prozeßgrößen $m_{G}, T_{L}$ und $T_{G}$ auf $\dot{m}_{V \text { vor }}$ vorsteuern läßt.

\subsection{Adaption der Durchflußmessung}

Wird $\dot{m}_{V}$ gemäß (19) vorgesteuert, stellt im stationären Zustand das Stellsignal des Temperaturreglers (I-Regler) einen Luftmassenstrom dar, der alle auftretenden Störgrößeneinflüsse repräsentiert. Neben Gasbeschaffenheitsänderungen stellt vor allem der mehr oder weniger systematische Fehler der Durchflußmessung eine derartige Störung dar. Mit der Kenntnis dieses Stellsignals wird eine Adaption der Kennlinie der Durchflußmessung durchgeführt. So wird bereits auf Basis der Durchflußregelung die gewünschte Verbrennungstemperatur eingestellt und ein lang andauernder Ausregelvorgang der überlagerten Temperaturregelung vermieden.

Ist der gemessene Luftmassenstrom $\dot{m}_{V \text { mess }}$ über einen längeren Zeitraum konstant, liegt ein stationärer Zustand vor, und das Stellsignal des I-Reglers wird als Stützstelle einer Fehlerfunktion $\varepsilon\left(\dot{m}_{V \text { mess }}\right)$ abgespeichert. So kann nach und nach - auch während des Betriebes - die gesamte Fehlerfunktion $\varepsilon\left(\dot{m}_{V, \text { mess }}\right)$ aufgenommen werden ${ }^{3}$. Die Subtraktion der so erhaltenen Fehlerkurve von den Meßwerten,

$$
\dot{m}_{V_{\text {adaptiert }}}=\dot{m}_{V, \text { mess }}-\varepsilon\left(\dot{m}_{V \text {, mess }}\right) \text {, }
$$

führt zu einem adaptierten Luftmassenstrom, der die gewünschte Kompensation der aufgetretenen Störungen bewirkt.

Das Gesamtkonzept der Regelung läßt sich zusammenfassend so charakterisieren (Bild 5): Der Verbrennungsluftmassenstrom wird über den vorgegebenen Gasmassenstrom vorgesteuert und von der nachge-

\footnotetext{
$\overline{3}$ Die Fehlerkurve $\varepsilon\left(\dot{m}_{V \text { mess }}\right)$ läßt sich im einfachsten Fall durch einen Polygonzug nachbilden.
}

schalteten Durchflußregelung schnell auf seinen Sollwert gebracht. Mit Hilfe einer sehr genauen $\lambda$-Messung des Abgasgemisches werden alle vorkommenden Störungen durch die überlagerte Temperaturregelung beseitigt. Aufgrund der begrenzten Dynamik des Temperaturregelkreises erstreckt sich ein solcher Ausregelvorgang über einen längeren Zeitraum und kann mit Hilfe einer Adaption der Durchflußmessung dauerhaft vermieden werden.

\section{Simulationen und praktische Erprobung}

Zur Überprüfung des Regelungskonzeptes und des Reglerentwurfs ist das Betriebsverhalten des geregelten Brennersystems in einem ersten Schritt auf einem Digitalrechner simuliert worden. Es konnte nachgewiesen werden, daß das Systemverhalten den gestellten Anforderungen genügt. Im zweiten Schritt wurde das Rcgelungssystem an einem Laborprüfstand mit einem Brennerprototyp experimentell untersucht. Dabei wurden die guten Simulationsergebnisse bestätigt. Parallel hierzu wurde das erstellte Prozeßmodell validiert.

Im folgenden werden zwei exemplarische Ergebnisse angegeben.

\subsection{Simulationen}

Zunächst wird die Betriebssituation ,Arbeitspunktrampe" betrachtet. Dabei wird angenommen, daß der Brenner während eines Zeitraums von $200 \mathrm{~s}$ linear von $30 \%$ auf $60 \%$ Last fährt. Bild 6 ist zu entnehmen, da 3 der Verbrennungsluftmassenstrom durch die schnelle unterlagerte Durchflußregelung praktisch ohne Verzögerung dem durch die Vorsteuerung berechneten Sollwert folgt. Trotzdem weicht die aus dem gemessenen $\lambda$-Wert berechnete Flammentemperatur zunächst von ihrem Sollwert $\left(1400^{\circ} \mathrm{C}\right) \mathrm{ab}$. Dies ist darauf zurückzuführen, daß mit Beginn der Arbeitspunktrampe die Temperatur der Verbrennungsluft ebenfalls linear zu steigen beginnt ${ }^{4}$, so daß gemäß (3) ein immer größer werdender $\lambda$-Wert zur Einhaltung der Solltemperatur zu fordern ist. Der von der Vorsteuerung korrekt eingestellte $\lambda$-Wert wird bedingt durch die Totzeit jedoch stets später gemessen und ,paßt" somit nicht zur momentanen Lufttemperatur. Die fälschlicherweise berechnete Temperaturdifferenz wird vom I-Regler der Temperaturregelung asymptotisch ausgeregelt, wodurch die tatsächliche Flammentemperatur etwas zu niedrig liegt. Nach Erreichen des stationären Arbeitspunktes tritt der gleiche Effekt in entgegengesetzter Richtung auf, wobei die Temperaturabweichung diesmal jedoch richtigerweise ausgeregelt wird. Unter der Voraussetzung langsamer Arbeitspunktwechsel hat dieses Verhalten der Regelung einen so geringen Einflu $B$ auf die Flammentemperatur, da $B$ es praktisch vernachlässigt werden kann.

\footnotetext{
${ }^{4}$ Der Temperaturverlauf ist durch prozeßspezifische Rahmenbedingungen vorgegeben.
} 

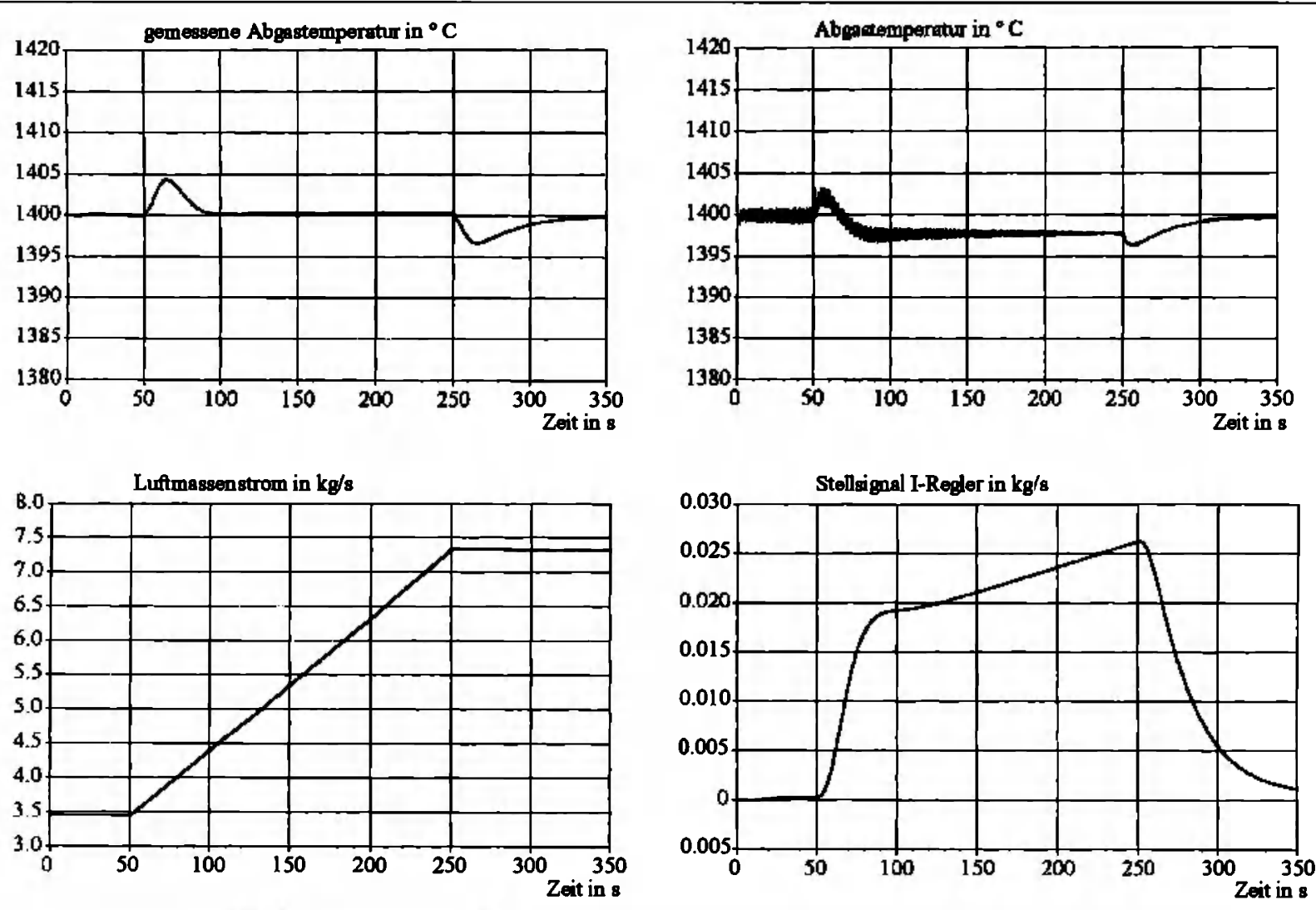

Bild 6: Simulationen zum Betriebsfall „Arbeitspunktrampe“.
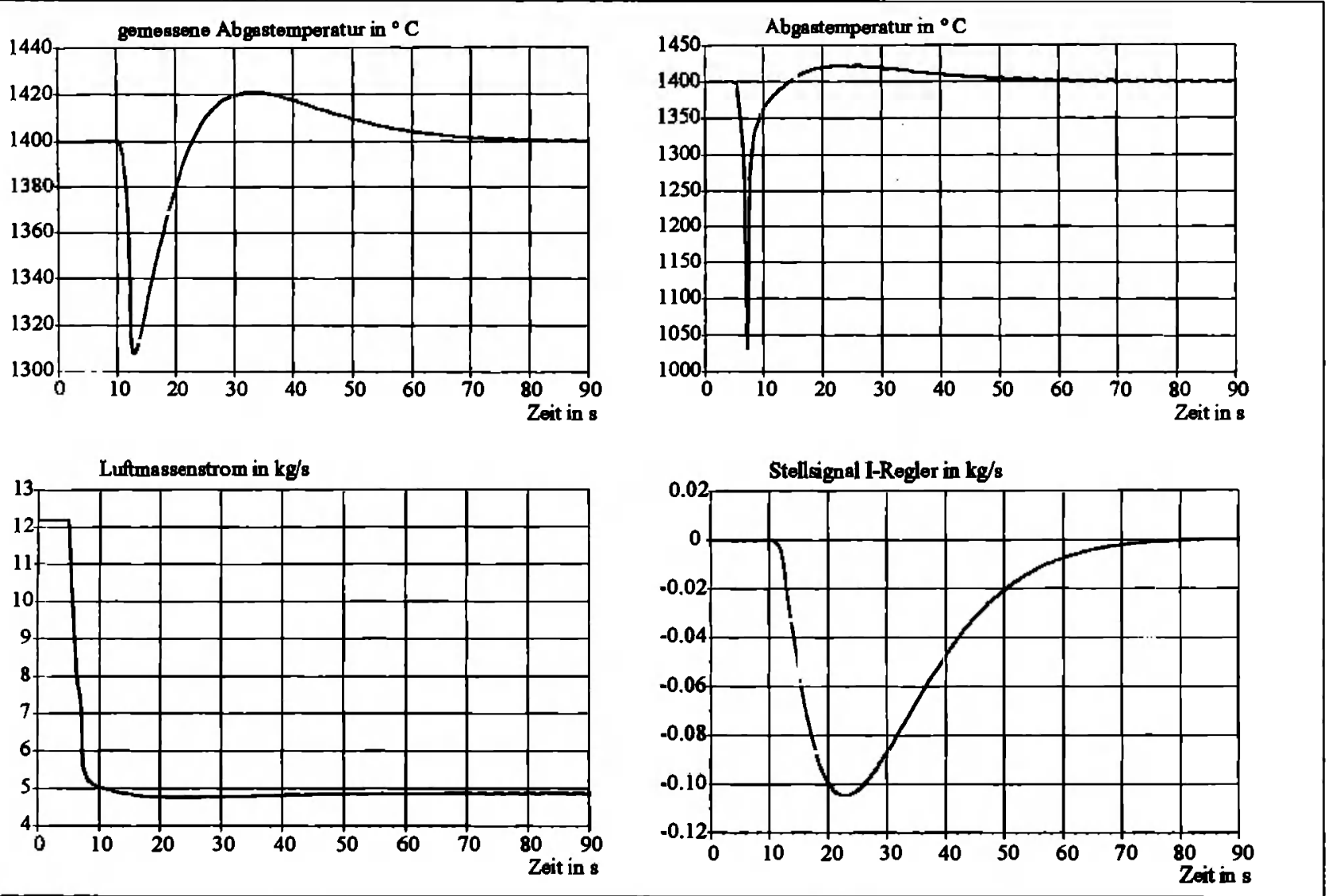

Bild 7: Simulationen zum Betriebsfall ,Lastabwurf:". 

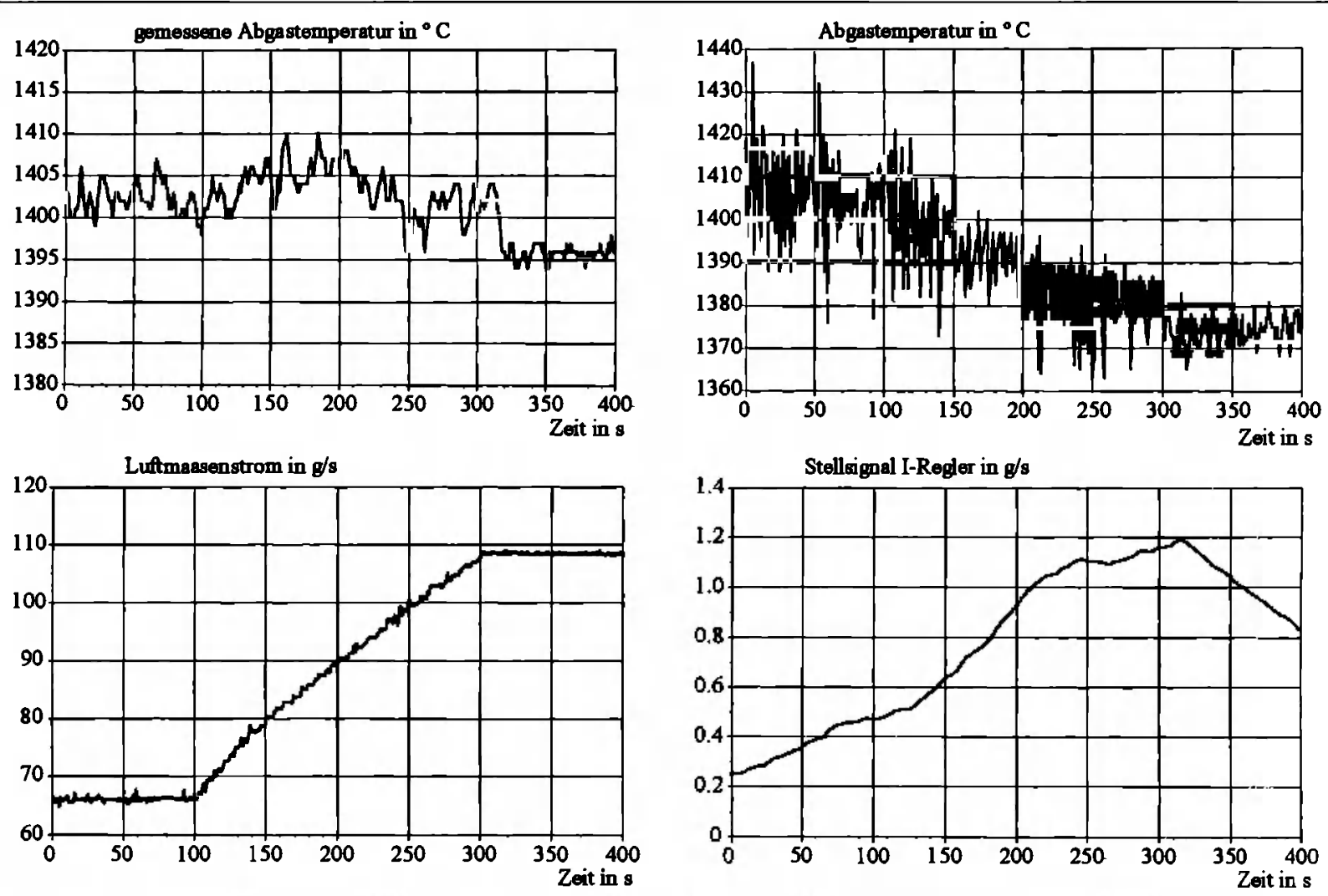

Bild 8: Praktische Erprobung des Betriebsfalls „Arbeitspunktrampe“
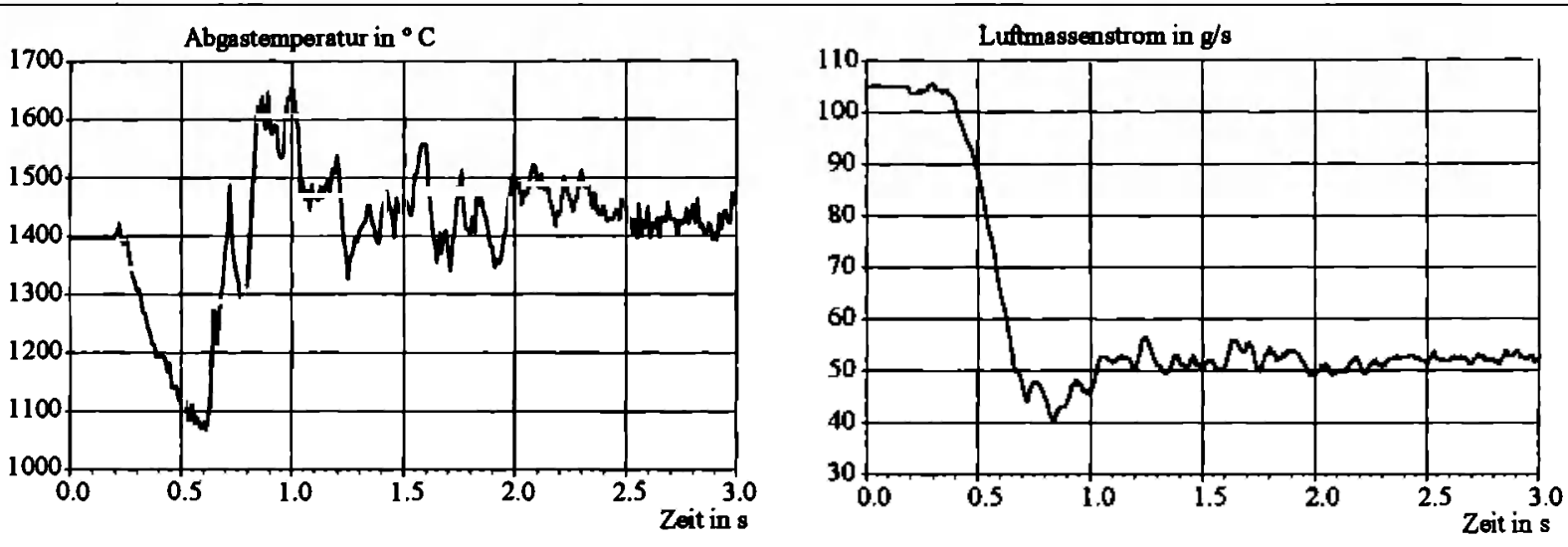

Bild 9: Praktische Erprobung des Betriebsfalls „Lastabwurf“.

Einen vergleichsweise kritischen Betriebsfall stellt der , Lastabwurf“ dar, bei dem der Gasmassenstrom zum Zeitpunkt $t_{0}=5 \mathrm{~s}$ innerhalb von $2 \mathrm{~s}$ linear von $100 \%$ auf $40 \%$ des Nennwertes abfällt. Bild 7 zeigt, $\mathrm{da} B$ aufgrund der begrenzten Dynamik des Durchflußkreises - hier sind in erster Hinsicht die Dynamiken der servohydraulischen Antriebseinheiten maßgebend - der Verbrennungsluftmassenstrom etwas länger als $2 \mathrm{~s}$ benötigt, um seinen neuen Sollwert zu erreichen. Dadurch sinkt die Flammentemperatur kurzfristig auf unter $1100^{\circ} \mathrm{C}$. Der sich anschließende Ausregelvorgang in der Flammentemperatur wird durch den Temperaturregler hervorgerufen, der den entstandenen Temperaturfehler mit der der $\lambda$-Messung eigenen Verzögerung aufintegriert.

\subsection{Praktische Erprobung}

Obwohl an dem Laborprüfstand nicht alle wichtigen Eigenschaften des Gasturbinenbrenners - wie z. B. die Dynamiken der Ventilantriebe - nachgebildet werden konnten, war es möglich, die wesentlichen Ergebnisse der Simulationen zu bestätigen.

Bild 8 zeigt, daß während des Verfahrens einer Arbeitspunktrampe die Flammentemperatur nahezu exakt auf ihrem Sollwert gehalten wird.

Auch im Experiment stellte sich der Lastabwurf als einziger kritischer Betriebsfall heraus. Er wurde aber von der Regelung ohne Probleme beherrscht (Bild 9). Unmittelbar nach dem Lastabwurf sinkt die Flammentemperatur zwar auf unter $1100^{\circ} \mathrm{C}$ ab, erreicht aber bereits $0.5 \mathrm{~s}$ später wieder ihren Sollwert. 


\section{Zusammenfassung und Ausblick}

In vorliegender Arbeit wurde ein Regelungskonzept vorgestellt, mit dem es möglich ist, extrem schadstoffarme, vorgemischte Gasturbinenbrennkammern beim Betrieb mit Erdgas mit niedrigsten $\mathrm{CO}-$ und $\mathrm{NO}_{\mathbf{x}}-$ Emissionen zu regeln. Diese Aufgabe läßt sich im wesentlichen auf die Konstanthaltung der Flammentemperatur bzw. des entsprechenden $\lambda$-Wertes zurückführen. Neben dieser primären Aufgabe war die Regelung aber auch so zu konzipieren, daß sie schnelle Änderungen - beispielsweise einen Lastabwurf - beherrschen kann, ohne daß aufgrund eines vorübergehenden Luftüberschusses die Flamme erlischt. Zur Lösung dieser Aufgabe wurde eine dreistufige Regelungsstruktur vorgesehen: Diese besteht zum einen aus einer schnellen, aber ungenauen Vorsteuerung und unterlagerten Regelung des Verbrennungsluftmassenstroms, des weiteren aus einer überlagerten Temperaturregelung, die zwar wesentlich langsamer, dafür aber sehr exakt ist, und schließlich aus einem Adaptionsteil, der mit Hilfe der Exaktheit der überlagerten Temperaturregelung die Genauigkeit der unterlagerten Regelung adaptiv verbessert.

$\mathrm{Da} ß$ das Regelungskonzept die gestellten Anforderungen auch tatsächlich erfüllt, konnte durch Simulationen und auch durch Versuche an einem Gasturbinenbrenner-Prototyp bestätigt werden. Damit sind die Voraussetzungen für die technische Implementierung der Regelung und ihren Einsatz in Verdichterstationen geschaffen. Darüber hinaus sind die Ergebnisse auch auf ähnliche Brennertypen übertragbar.

\section{Danksagung}

Die Autoren danken Herrn Prof. Dr.-Ing. R. Tracht, Frau Dipl.-Ing. U. Krahmann sowie den Herren Dipl.-Ing. W. Heinrichs und Dipl.Ing. R. Struhalla für fruchtbare Diskussionen und ihre Unterstützung bei der Durchführung der Simulationen.

\section{Literatur}

[1] Altemark, D.: Feuerungskonzepte für Gasturbinen. Die Industriefeuerung, Heft 55, S. 38-47, 1992.

[2] Saul, A. und Altemark, D.: Lean-burn premixed combustion in gas turbine combusters. GASWÄRME International Band 40, Heft 7/8, S. 336-342, 1991.

[3] Altemark, D. und Knauber, R.: Ergebnisse von Untersuchungen an einem Vormischbrenner unter Druck mit extrem niedriger $\mathrm{NO}_{\mathrm{x}}$-Emission. VDI-Berichte $\mathrm{Nr}$. 645, S. 299-311, 1987.

[4] Seifert, G.E.: Ein Beitrag zur experimentellen Untersuchung der Verbrennung und der Schadstoffbildung in Gasturbinenbrennkammern. Dissertation, Fortschritt-Berichte VDI Reihe 6, Nr.188, VDI-Verlag 1986.

[5] Altemark, D. und Hess, R.: Eine neue Brennergeneration für Luftreinhaltung und Energieeinsparung. GASWÄRME International Band 32, Heft 1, S. 40-46, 1983.

[6] Baehr, H.D.: Thermodynamik. Springer-Verlag 1987.

[7] Becker, E.: Technische Strömungslehre. Teubner-Verlag 1986.

[8] Bohl, N.: Technische Strömungslehre. Vogel-Verlag 1980.

[9] MOOG GmbH: Catalog 771 1281. Datenblätter zu MOOGServoventilen der Baureihe D770, Böblingen 1990.

[10] Faulhaber, S.: Lageregelungen für hydraulische Servoantriebe. Fortschritt-Berichte VDI Reihe 8 Nr. 84, VDI-Verlag 1985.

[11] Rohr, F. J.: Sensor zur Messung und Regelung von Sauerstoffkonzentationen bei Verbrennungsprozessen. Messen + Prüfen $1 / 2,1984$.

[12] Egner, M.: Hochdynamische Lageregelung mit elektrohydraulischen Antrieben. ISW Forschung und Praxis Band 74, Springer-Verlag 1988.

[13] Roth, J.: Regelungskonzepte für lagegeregelte elektrohydraulische Servoantriebe. Dissertation, RWTH Aachen 1983.

Manuskripteingang: 11. Dezember 1995

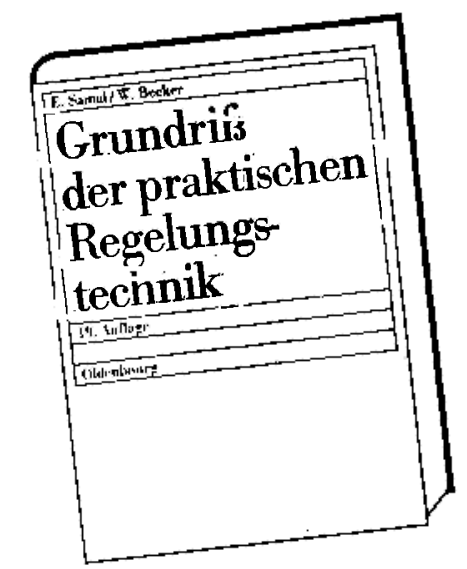

Das Standardwerk für jeden Praktiker bereits die 19. Auflage!

Im Gegensatz zu mathematisch orientierten Darstellungen, stellt dieses Werk die physikalisch-technischen Zusammenhänge und die praktische Regelungstechnik in den Vordergrund. Alle wesentlichen Fragen der praktischen - analogen und digitalen - Regelungstechnik sind behandelt; zahlreiche der Praxis entnommene Beispiele illustrieren die Verfahren.
Erwin Samal/Wilhelm Becker

\section{Grundrif der praktischen Regelungstechnik}

19 , verbesserte und überarbeitete Auflage 1996. 604 Seiten, 320 Abbildungen,

32 Tabellen,

DM 49,80/öS 369,-/sFr 49,80

ISBN 3-486-23635-0

Oldenbourg 